\title{
Solving delay differential equations of pantograph type using predictor-corrector method
}

\begin{abstract}
In this paper, the fourth order predictor-corrector method in the form of linear multistep method will be presented to solve delay differential equations of pantograph type. This special type of delay differential equations always has the delay term fall after the initial value but before the desire approximation being calculated. The new approach will be discussed on how to calculate the delay term which is needed in the approximation. The implementation is based on the predictor-corrector formulas in the PECE mode and the proposed method will approximate the solutions using constant step size. Numerical results are presented to show the efficiency of the proposed method.
\end{abstract}

Keyword: Delay differential equations; Pantograph type; Predictor-corrector method 